\title{
Some results on the size of sum and product sets of finite sets of real numbers
}

\author{
Derrick Hart and Alexander Niziolek \\ (Communicated by Andrew Granville)
}

Let $A$ and $B$ be finite subsets of positive real numbers. Solymosi gave the sumproduct estimate $\max (|A+A|,|A \cdot A|) \geq(4\lceil\log |A|\rceil)^{-1 / 3}|A|^{4 / 3}$, where \lceil\rceil is the ceiling function. We use a variant of his argument to give the bound

$$
\max (|A+B|,|A \cdot B|) \geq(4\lceil\log |A|\rceil\lceil\log |B|\rceil)^{-1 / 3}|A|^{2 / 3}|B|^{2 / 3} .
$$

(This isn't quite a generalization since the logarithmic losses are worse here than in Solymosi's bound.)

Suppose that $A$ is a finite subset of real numbers. We show that there exists an $a \in A$ such that $|a A+A| \geq c|A|^{4 / 3}$ for some absolute constant $c$.

\section{Introduction}

Given finite subsets $A$ and $B$ of an additive group, the sum set of $A$ and $B$ is

$$
A+B=\{a+b: a \in A, b \in B\} .
$$

Similarly, define the product set by

$$
A \cdot B=\{a b: a \in A, b \in B\} .
$$

If $M$ and $N$ are numbers (depending on $A$ and $B$ ) we write $M \gtrsim N$ to mean that $M \geq c N$ for some constant $c>0$ (independent of $A$ and $B$ ). We write $M \approx N$ to mean that $c N \leq M \leq c^{\prime} N$ for $c, c^{\prime}>0$.

Suppose that $A=B$ is an arithmetic progression. Then

$$
|A+A| \lesssim|A| \text { and }|A \cdot A| \gtrsim|A|^{2-\delta},
$$

where here and throughout $\delta \rightarrow 0$ as $|A| \rightarrow \infty$ and $|\cdot|$ denotes the size of the set. In contrast, if $A=B$ is a geometric progression then

$$
|A+A| \gtrsim|A|^{2} \text { and }|A \cdot A| \lesssim|A| .
$$

MSC2000: 11B13, 11B75.

Keywords: sum-product estimate, multiplicative energy, Solymosi bound. 
These examples led Erdős and Szemerédi [1983] to ask whether both the product set and sum set can be small at the same time. They conjectured that it is not possible in the following sense.

Conjecture 1. Let $A$ be a finite subset of $\mathbb{Z}$. Then

$$
\max (|A+A|,|A \cdot A|) \geq|A|^{2-\delta} .
$$

They showed that

$$
\max (|A+A|,|A \cdot A|) \geq|A|^{1+\varepsilon},
$$

for a positive $\varepsilon$.

The explicit bound $\varepsilon \geq \frac{1}{31}$ was given by Nathanson [1997], and $\varepsilon \geq \frac{1}{15}$ by Ford [1998]. A breakthrough was achieved by Elekes [1997], who connected the problem to incidence geometry and applied the Szemerédi-Trotter incidence theorem to obtain $\varepsilon \geq \frac{1}{4}$. This was improved by Solymosi [2005] to $\varepsilon \geq \frac{3}{14}-\delta$. These bounds hold in the more general context of finite subsets of $\mathbb{R}$.

Recently, by a short and ingenious argument it was shown by Solymosi [2009] that $\varepsilon \geq \frac{1}{3}-\delta$. In Section 3 we mimic Solymosi's argument with a few changes to give an analogous estimate for sums and products of different sets.

Given the strong relationship between sums and products one may ask a related question: how large is the set $A \cdot B+C$ guaranteed to be? Elekes (see [Alon and Spencer 2000]) showed that $|A \cdot B+C| \gtrsim \sqrt{|A||B||C|}$ with certain size restrictions on the three sets. His argument relied on the aforementioned Szeremedi-Trotter incidence theorem and is short enough to present in the next few lines.

Let $P$ be a set of points in $\mathbb{R}^{2}$ and $L$ a set of lines. We say a point $p \in P$ is incident to a line $l \in L$ if $p$ lies on $l$. In this case, we denote this incidence by $(p, l) \in P \times L$.

Theorem 2 [Szemerédi and Trotter 1983]. Let $I_{P, L}$ denote the number of incidences between $P$ and $L$. Then bound

$$
I_{P, L} \lesssim|P|^{2 / 3}|L|^{2 / 3}+|P|+|L| .
$$

Let $L=\{y=a x+c: a \in A, c \in C\}$ and $P=B \times A \cdot B+C$. Clearly, given any $a \in A, b \in B, c \in C$, the point $(b, a b+c)$ is incident to the line $y=a x+c$. Therefore, by Szemeredi-Trotter, $|A||B||C| \lesssim|A|^{2 / 3}|B|^{2 / 3}|C|^{2 / 3}|A \cdot B+C|^{2 / 3}$.

In the context of $\mathbb{F}_{q}$, the finite field containing $q$ elements, similar questions have been explored as well. Bourgain [2005] showed that for $A \subseteq \mathbb{F}_{q}$ such that $|A| \gtrsim q^{3 / 4}$, one has $A \cdot A+A \cdot A+A \cdot A=\mathbb{F}_{q}$; in particular, if $|A| \approx q^{3 / 4}$, then $|A \cdot A+A \cdot A+A \cdot A| \gtrsim|A|^{4 / 3}$. In [Hart and Iosevich 2008] it was shown that if $|A| \gtrsim q^{3 / 4}$, then $A \cdot A+A \cdot A=\mathbb{E}_{q}^{*}$; in particular, if $|A| \approx q^{3 / 4}$, then $|A \cdot A+A \cdot A| \gtrsim|A|^{4 / 3}$. Due to the misbehavior of the zero element, it is not possible to guarantee that $A \cdot A+A \cdot A=\mathbb{F}_{q}$ unless $A$ is a positive proportion of 
the elements of $\mathbb{F}_{q}$. Under the weaker conclusion that $|A \cdot A+A \cdot A| \gtrsim q$ it is shown in the same paper that one may take $|A| \gtrsim q^{2 / 3}$. Shparlinski [2008] applied multiplicative character sums to show that if $|A| \gtrsim q^{2 / 3}$, then $|A \cdot A+A| \gtrsim q$, implying that if $|A| \approx q^{2 / 3}$, then $|A \cdot A+A| \gtrsim|A|^{3 / 2}$.

Theorem 3 [Chapman et al. 2009, Theorem 2.10]. Let A be a subset of $\mathbb{F}_{q}^{*}$. Then

$$
|A|^{-1} \sum_{a \in A}|a A+A| \gtrsim \min \left(q,|A|^{3} q^{-1}\right) .
$$

In particular, if $|A| \approx q^{2 / 3}$, there exists a subset $A^{\prime}$ of $A$ with $\left|A^{\prime}\right| \gtrsim|A|$ such that

$$
|a A+A| \gtrsim|A|^{3 / 2} \approx q,
$$

for all $a \in A^{\prime}$.

It is natural to ask whether a similar statement holds in the case that $A$ is a finite subset of the real numbers. We show that this is in fact the case in Section 4.

\section{Statement of results}

Define the multiplicative energy of the finite subsets $A, B, C, D$ of real numbers by

$$
\mathrm{E}(A, B, C, D)=\left|\left\{\left(x_{1}, x_{2}, y_{1}, y_{2}\right) \in A \times B \times C \times D: x_{1} y_{2}=x_{2} y_{1}\right\}\right| .
$$

For $A, B$ finite subsets of positive real numbers with $|A| \leq|B|$, the argument of [Solymosi 2009] gives the bound

$$
\mathrm{E}(A, B, A, B) \leq 4\lceil\log |A|\rceil|A+A||B+B| .
$$

A short Cauchy-Schwarz argument gives that $\mathrm{E}(A, B, A, B) \geq|A|^{2}|B|^{2} /|A \cdot B|$, which in turn gives the sum-product inequality

$$
|A|^{2}|B|^{2} \leq 4\lceil\log |A|\rceil|A+A||B+B||A \cdot B| .
$$

In the case that $A=B$, this immediately implies the Solymosi sum-product bound discussed in the introduction:

$$
\max (|A+A|,|A \cdot A|) \geq(4\lceil\log |A|\rceil)^{-1 / 3}|A|^{4 / 3} .
$$

We will use a slight variant of the argument of Solymosi to give a different bound on the multiplicative energy:

Theorem 4. Let $A, B, C, D$ be finite subsets of positive real numbers. Then

$$
\mathrm{E}(A, B, C, D) \leq 4\lceil\log (\min (|A|,|C|))\rceil\lceil\log (\min (|B|,|D|))\rceil|A+B||C+D| .
$$


(Notice that the logarithmic loss is worse than what was obtained by Solymosi.)

Using the fact that $\mathrm{E}(A, B, A, B) \geq|A|^{2}|B|^{2} /|A \cdot B|$, we obtain the following sum-product estimate.

Corollary 5. Let A, B be finite subsets of positive real numbers. Then

$$
\max (|A+B|,|A \cdot B|) \geq(4\lceil\log |A|\rceil\lceil\log |B|\rceil)^{-1 / 3}|A|^{2 / 3}|B|^{2 / 3} .
$$

One may compare this to the result of applying Plünnecke's inequality to (2-2):

$$
\max (|A+B|,|A \cdot B|) \geq(4\lceil\log |A|\rceil)^{-1 / 5}|A|^{3 / 5}|B|^{3 / 5} .
$$

We will also show this:

Theorem 6. Let $A, B, C$ be finite subsets of $\mathbb{R}$ such that $|B|^{1 / 2}|C|^{-1 / 2} \lesssim|A| \lesssim$ $|B|^{2}|C|$. Then

$$
|A|^{-1} \sum_{a \in A}|a B+C| \gtrsim|A|^{1 / 3}|B|^{1 / 3}|C|^{2 / 3} .
$$

In particular, there exists an $a \in A$ such that

$$
|a B+C| \gtrsim|A|^{1 / 3}|B|^{1 / 3}|C|^{2 / 3} .
$$

\section{Proof of Theorem 4}

We begin by writing

$$
\begin{aligned}
\mathrm{E}(A, B, C, D) & =\sum_{x_{1} y_{2}=x_{2} y_{1}} A\left(x_{1}\right) B\left(x_{2}\right) C\left(y_{1}\right) D\left(y_{2}\right) \\
& =\sum_{t \neq 0} \sum_{\substack{x_{1}=t x_{2} \\
y_{1}=t y_{2}}}(A \times C)\left(x_{1}, y_{1}\right)(B \times D)\left(x_{2}, y_{2}\right),
\end{aligned}
$$

where $A(\cdot)$ denotes the characteristic function of the set $A$ and $\times$ denotes the Cartesian product. Summing in $t$ we have

$$
\mathrm{E}(A, B, C, D)=\sum_{y \in(B \times D)}\left|(A \times C) \cap l_{m_{y}}\right|
$$

where $l_{m_{y}}$ is the line through the origin and the point $y$ with slope $m_{y}$. Each $y \in(B \times D)$ lies on some line $l_{m_{y}}$ with $m_{y} \in D \cdot B^{-1}=\left\{d b^{-1}: d \in D, b \in B\right\}$. Since the quantity $\left|(A \times C) \cap l_{m_{y}}\right|$ is constant and nonzero for each $y$ on $l_{m_{y}}$ with slope $m_{y}$ in $C \cdot A^{-1}$, we have

$$
\mathrm{E}(A, B, C, D)=\sum_{m \in M}\left|(A \times C) \cap l_{m}\right|\left|(B \times D) \cap l_{m}\right|,
$$


where $M=C \cdot A^{-1} \cap D \cdot B^{-1}$. We then take a dyadic decomposition

$$
\mathrm{E}(A, B, C, D)=\sum_{\substack{0 \leq i \leq\lceil\log (\min (|A|,|C|))\rceil \\ 0 \leq j \leq\lceil\log (\min (|B|,|D|))\rceil}} \sum_{m \in M_{i, j}}\left|(A \times C) \cap l_{m}\right|\left|(B \times D) \cap l_{m}\right|,
$$

where $M_{i, j}=\left\{m \in M: 2^{i} \leq\left|(A \times C) \cap l_{m}\right|<2^{i+1}, 2^{j} \leq\left|(B \times D) \cap l_{m}\right|<2^{j+1}\right\}$. Therefore, for some $i^{\prime}$ and $j^{\prime}$,

$$
\frac{\mathrm{E}(A, B, C, D)}{\lceil\log (\min (|A|,|C|))\rceil\lceil\log (\min (|B|,|D|))\rceil} \leq \sum_{m \in M_{i^{\prime}, j^{\prime}}}\left|(A \times C) \cap l_{m}\right|\left|(B \times D) \cap l_{m}\right| .
$$

Set $n=\left|M_{i^{\prime}, j^{\prime}}\right|$ and order the elements of $M_{i^{\prime}, j^{\prime}}: m_{1}<m_{2}<\ldots<m_{n}$. This gives

$$
\frac{\mathrm{E}(A, B, C, D)}{\lceil\log (\min (|A|,|C|))\rceil\lceil\log (\min (|B|,|D|))\rceil} \leq 4 n 2^{i^{\prime}+j^{\prime}} .
$$

Given that $\left|(A \times C) \cap l_{m_{l}}+(B \times D) \cap l_{m_{l+1}}\right|=\left|(A \times C) \cap l_{m_{l}}\right|\left|(B \times D) \cap l_{m_{l+1}}\right|$, noting that any two sum sets $(A \times C) \cap l_{m_{l}}+(B \times D) \cap l_{m_{l+1}}$ and $(A \times C) \cap l_{m_{k}}+(B \times D) \cap l_{m_{k+1}}$ are disjoint for any $l \neq k$ gives

$$
n 2^{i^{\prime}+j^{\prime}} \leq\left|\bigcup_{l=1}^{n}\left((A \times C) \cap l_{m_{l}}+(B \times D) \cap l_{m_{l+1}}\right)\right| \leq|A+B||C+D| .
$$

Here, in an abuse of notation, $(B \times D) \cap l_{m_{n+1}}$ is the orthogonal projection of $(B \times D) \cap l_{m_{n}}$ onto the vertical line running through the minimal element of $B$. We may without loss of generality assume that the minimal element of $B$ is also the minimal element of $A \cup B$.

\section{Proof of Theorem 6}

We will need a lemma, whose proof we will delay until the end of the section.

Lemma 7. Let $A, B, C$ be finite subsets of $\mathbb{R}$ such that $|B|^{1 / 2}|C|^{-1 / 2} \lesssim|A| \lesssim$ $|B|^{2}|C|$. Then

$|\{(a, b, c, d, e) \in A \times B \times C \times B \times C: a b+c=a d+e\}| \lesssim|A|^{2 / 3}|B|^{5 / 3}|C|^{4 / 3}$.

With this lemma in hand one may then apply the Cauchy-Schwarz inequality:

$$
\begin{aligned}
& |A||B|^{2}|C|^{2}=|A|^{-1}\left(\sum_{\substack{t \in a B+C \\
a \in A}} \sum_{a b+c=t} B(b) C(c)\right)^{2} \\
& \quad \leq\left(|A|^{-1} \sum_{a \in A}|a B+C|\right) \sum_{\substack{t \in a B+C \\
a \in A}}\left(\sum_{a b+c=t} B(b) C(c)\right)^{2} .
\end{aligned}
$$


Noting that

$$
\begin{aligned}
\sum_{\substack{t \in a B+C \\
a \in A}}\left(\sum_{a b+c=t} B(b) C(c)\right)^{2} & \\
& =|\{(a, b, c, d, e) \in A \times B \times C \times B \times C: a b+c=a d+e\}|
\end{aligned}
$$

completes the proof of Theorem 6 .

Proof of Lemma 7. We will apply the Szemerédi-Trotter incidence theorem. For a fixed $b \in B$, consider the set of lines $L_{b}=\{y=(b-d) x+c: c \in C, d \in B\}$. Also consider the set of points $P=\{(a, e) \in(A \times C)\}$. Then $\mid\{(a, b, c, d, e) \in$ $A \times B \times C \times B \times C: a b+c=a d+e\}|\leq| B \mid \max _{b \in B} I_{P, L_{b}}$. Noting that $\left|L_{b}\right|=|B||C|$ and $|P|=|A||C|$ and applying the Szemerédi-Trotter theorem gives

$|\{(a, b, c, d, e) \in A \times B \times C \times B \times C: a b+c=a d+e\}| \lesssim|A|^{2 / 3}|B|^{5 / 3}|C|^{4 / 3}$, as long as $|B|^{1 / 2}|C|^{-1 / 2} \lesssim|A| \lesssim|B|^{2}|C|$.

\section{Remarks}

The argument of Elekes [1997] actually gives a more general bound for finite subsets $A, B, C$ of positive real numbers:

$$
\max (|A+B|,|A \cdot C|) \gtrsim|A|^{3 / 4}|B|^{1 / 4}|C|^{1 / 4} .
$$

A direct application of Plünnecke's inequality [Tao and Vu 2006, Corollary 6.26] to $(2-3)$ gives

$$
\max (|A+B|,|A \cdot C|) \geq(4\lceil\log |A|\rceil)^{-1 / 6}|A|^{2 / 3}|B|^{1 / 3}|C|^{1 / 6} .
$$

This bound is preferable if $|B|$ is much larger than $|A||C|$. We do not currently know of a way to use Solymosi's argument to obtain an improved bound for the case that the three sets are close together in size.

\section{References}

[Alon and Spencer 2000] N. Alon and J. H. Spencer, The probabilistic method, 2nd ed., WileyInterscience, New York, 2000. MR 2003f:60003 Zbl 0996.05001

[Bourgain 2005] J. Bourgain, "Mordell's exponential sum estimate revisited", J. Amer. Math. Soc. 18:2 (2005), 477-499. MR 2006b:11099 Zbl 1072.11063

[Chapman et al. 2009] J. Chapman, M. B. Erdoğan, D. Hart, A. Iosevich, and D. Koh, "Pinned distance sets, $k$-simplices, Wolff's exponent in finite fields and sum-product estimates", preprint, 2009. arXiv 0903.4218

[Elekes 1997] G. Elekes, "On the number of sums and products", Acta Arith. 81:4 (1997), 365-367. MR 98h:11026 Zbl 0887.11012 
[Erdős and Szemerédi 1983] P. Erdôs and E. Szemerédi, “On sums and products of integers”, pp. 213-218 in Studies in pure mathematics, Birkhäuser, Basel, 1983. MR 86m:11011 Zbl 0526.10011

[Ford 1998] K. Ford, "Sums and products from a finite set of real numbers", Ramanujan J. 2:1-2 (1998), 59-66. MR 99i:11014 Zbl 0908.11008

[Hart and Iosevich 2008] D. Hart and A. Iosevich, "Sums and products in finite fields: an integral geometric viewpoint", pp. 129-135 in Radon transforms, geometry, and wavelets, Contemp. Math. 464, Amer. Math. Soc., Providence, RI, 2008. MR 2009m:11032

[Nathanson 1997] M. B. Nathanson, "On sums and products of integers", Proc. Amer. Math. Soc. 125:1 (1997), 9-16. MR 97c:11010 Zbl 0869.11010

[Shparlinski 2008] I. E. Shparlinski, "On the solvability of bilinear equations in finite fields", Glasg. Math. J. 50:3 (2008), 523-529. MR 2009j:11189 Zbl 05350503

[Solymosi 2005] J. Solymosi, "On the number of sums and products", Bull. London Math. Soc. 37:4 (2005), 491-494. MR 2006c:11021 Zbl 1092.11018

[Solymosi 2009] J. Solymosi, "Bounding multiplicative energy by the sumset", Adv. Math. 222:2 (2009), 402-408. MR 2538014 Zbl 05597906

[Szemerédi and Trotter 1983] E. Szemerédi and W. T. Trotter, Jr., "Extremal problems in discrete geometry”, Combinatorica 3:3-4 (1983), 381-392. MR 85j:52014 Zbl 0541.05012

[Tao and Vu 2006] T. Tao and V. Vu, Additive combinatorics, vol. 105, Cambridge University Press, Cambridge, 2006. MR 2008a:11002 Zbl 1127.11002

Received: 2009-09-07 Accepted: 2009-11-12

dnhart@math.rutgers.edu

alexnizi@eden.rutgers.edu
Department of Mathematics, Rutgers University, Piscataway, NJ 08854-8019, United States http://www. math.rutgers.edu/ dnhart/

School of Engineering, Rutgers University, Piscataway, NJ 08854-8019, United States 